\title{
BP-BEST-OP-2
}

\section{Multi-biomarker panel prediction model for diagnosis of pancreatic cancer}

Doo-Ho LEE ${ }^{1,2}$, Woongchang YOON ${ }^{3}$, Areum LEE', Youngmin HAN', Yoonhyeong BYUN', Jae Seung KANG', Hongbeom KIM', Wooil KWON', Young-Ah SUH', Yonghwan $\mathrm{CHOI}^{4}$, Junghyun NAMKUNG ${ }^{4}$, Sangjo HAN ${ }^{4}$, Sung Gon $\mathrm{YI}^{4}$, Jin Seok HEO ${ }^{5}$, In Woong HAN ${ }^{5}$, Joon Oh PARK ${ }^{6}$, Joo Kyung PARK ${ }^{6,7}$, Song Cheol KIM ${ }^{8}$, Eunsung JUN ${ }^{8}$, Chang Moo KANG ${ }^{9}$, Woo Jin LEE ${ }^{10}$, Hyeon Kook LEE" Huisong LEE ${ }^{11}$, Seungyeoun LEE ${ }^{12}$, Seung-Yong JEONG', Kyu Eun LEE', Wonshik HAN', Taesung PARK', Jin-Young JANG*1

'Department of Surgery and Cancer Research Institute, College of Medicine, Seoul National University, Seoul, Korea

${ }^{2}$ Department of Surgery, Gachon University Gil Medical Center, Incheon, Korea

${ }^{3}$ Bio-MAX/N-Bio Institute, Seoul National University, Seoul, Korea

${ }^{4}$ Immunodiagnostics R\&D Team, IVD Business Unit 5, SK Telecome, Seoul, Korea

${ }^{5}$ Department of Surgery, Samsung Medical Center, Sungkyunkwan University School of Medicine, Seoul, Korea

${ }^{6}$ Department of Internal Medicine, Samsung Medical Center, Sungkyunkwan University School of Medicine, Seoul, Korea

'Department of Health Sciences and Technology, SAIHST, Sungkyunkwan University, Seoul, Korea

${ }^{8}$ Department of Surgery, University of Ulsan College of Medicine and Asan Medical Center, Seoul, Korea

${ }^{9}$ Department of Surgery, Severance Hospital, Yonsei University College of Medicine, Seoul, Korea

${ }^{10}$ Center for Liver Cancer, National Cancer Center, Seoul, Korea

${ }^{11}$ Department of Surgery, Ewha Womans University School of Medicine, Seoul, Korea

${ }^{12}$ Department of Mathematics and Statistics, Sejong University, Seoul, Korea

${ }^{13}$ Department of Statistics, Seoul National University, Seoul, Korea

Introduction: The current study aimed to develop a prediction model using a multi-marker panel as a diagnostic screening tool for pancreatic-ductal-adenocarcinoma.

Methods: Multi-center cohort of 1,991 blood samples were collected from January 2011 to September 2019, of which 609 are normal, 145 are other-cancer (colorectal, thyroid, and breast cancer), 314 are pancreatic-benign-disease, and 923 are pancreatic-ductal-adenocarcinoma. The automated multi-biomarker Enzyme-Linked Immunosorbent Assay kit was developed using three potential biomarkers, LRG1, TTR, and CA 19-9. Using a logistic regression model trained on training data set, the predicted values for pancreatic-ductal-adenocarcinoma were obtained, and the result was classified into one of the three risk groups: low, intermediate, and high. The five covariates used to create the model were sex, age, and three biomarkers.

Results: Participants were categorized into four groups as normal $(\mathrm{n}=609)$, other-cancer $(\mathrm{n}=145)$, pancreatic-benign-disease $(\mathrm{n}=$ $314)$, and pancreatic-ductal-adenocarcinoma $(\mathrm{n}=923)$. The normal, other-cancer, and pancreatic-benign-disease groups were clubbed into the non-pancreatic-ductal-adenocarcinoma group $(\mathrm{n}=1,068)$. The positive and negative predictive value, sensitivity, and specificity were $94.12,90.40,93.81$, and 90.86 , respectively.

Conclusions: This study demonstrates a significant diagnostic performance of the multi-marker panel in distinguishing pancreatic-ductal-adenocarcinoma from normal and benign pancreatic disease states, as well as patients with other cancers. The model satisfies the requirements of an ideal screening test, being simple to use, less expensive, and having a good diagnostic efficacy with NPV, PPV, Sen, and Spe, all greater than $90.0 \%$. 